Cockcroft-11-02

Beam tomography in transverse normalised phase space

K.M. Hock ${ }^{\text {a,c, }}$, M.G.Ibison ${ }^{\text {a,c }}$, D.J.Holder ${ }^{\text {a,c }}$, A.Wolski ${ }^{\text {a,c }}$, B.D.Muratori ${ }^{\text {b,c }}$

${ }^{a}$ Department of Physics, University of Liverpool, Liverpool L69 7ZE, UK ${ }^{\mathrm{b}}$ ASTeC, STFC Daresbury Laboratory, Warrington WA4 4AD, UK

${ }^{\mathrm{C}}$ Cockcroft Institute, Daresbury Science and Innovation Campus, Daresbury, Warrington WA4 4AD, UK 


\title{
Beam tomography in transverse normalised phase space ${ }^{\text {is }}$
}

\author{
K.M. Hock ${ }^{\mathrm{a}, \mathrm{c}, *}$, M.G. Ibison ${ }^{\mathrm{a}, \mathrm{c}}$, D.J. Holder ${ }^{\mathrm{a}, \mathrm{c}}$, A. Wolski ${ }^{\mathrm{a}, \mathrm{c}}$, B.D. Muratori ${ }^{\mathrm{b}, \mathrm{c}}$ \\ a Department of Physics, University of Liverpool, Liverpool L69 7ZE, United Kingdom \\ ${ }^{\mathrm{b}}$ ASTeC, STFC Daresbury Laboratory, Warrington WA4 4AD, United Kingdom \\ ${ }^{\mathrm{c}}$ Cockcroft Institute, Daresbury Science and Innovation Campus, Daresbury, Warrington WA4 4AD, United Kingdom
}

\section{A R T I C L E I N F O}

\section{Article history:}

Received 11 January 2011

Received in revised form

7 March 2011

Accepted 3 April 2011

Keywords:

Tomography

Phase space

Electron beam

\begin{abstract}
A B S T R A C T
We demonstrate that the normalised phase space can be used to improve the quality of the reconstructed image in phase space tomography. This study uses the Filtered Back Projection (FBP) for reconstruction. We simulate the tomographic projections of a Gaussian phase space distribution in real phase space and in normalised phase space. The reconstruction can be carried out in real phase space directly. It can also be done first in normalised phase space, and then the co-ordinates can be transformed to real phase space. Using FBP, we show that the latter procedure produces images that agree well with the original distribution even when there are only three angles. It also gives better resolution for a more complex distribution, which we simulate with a random collection of Gaussian spots.
\end{abstract}

c) 2011 Published by Elsevier B.V.

\section{Introduction}

Beam tomography is widely used to characterise the properties of a beam of charged particles [1-6]. By recording the images of the cross-section of a beam as it undergoes a sequence of transformations in phase space, the phase space distribution of the beam can be reconstructed using standard algorithms [9-11]. As a beam of electrons progresses through a beamline, the phase space distribution of the beam changes as a result of the dynamical effects of the lattice magnets and drift spaces. Reconstructing the phase space usually means determining the phase space at a particular position in the beamline. In the designs of some accelerators, screens have been incorporated in sections of the beamlines for tomography measurements. Various methods have been used to provide suitable phase space transformations of the beam at the screens. These methods include changing the strengths of a solenoid [3,7,8], changing the strengths of a quadrupole [1], and simply allowing the beam to propagate through a drift space [12,13].

As an example, the tomography section at PITZ [12] includes four screens for measuring the transverse profile of the beam. The positions of the screens are designed such that the betatron phase advance (horizontally and vertically) between adjacent screens is $45^{\circ}$. A similar system is used at the FLASH [14]. In the tomography section at ALICE [13], three screens are placed at positions with

Work supported by the Science and Technology Facilities Council, UK.

* Corresponding author at: Department of Physics, University of Liverpool, Q2 Liverpool L69 7ZE, United Kingdom.

E-mail address: kai.hock@stfc.ac.uk (K.M. Hock). intervals of $60^{\circ}$ in betatron phase advance. The betatron phase advances are determined by the Twiss parameters, as defined in the original design of the beamline. The work in this paper is motivated by the observation that this phase advance corresponds to rotation angles in the normalised phase space. In the absence of betatron coupling, the horizontal, transverse normalised phase space is defined by the following transformation:

$\left(\begin{array}{l}x_{N} \\ x_{N}^{\prime}\end{array}\right)=\left(\begin{array}{cc}\frac{1}{\sqrt{\beta}} & 0 \\ \frac{\alpha}{\sqrt{\beta}} & \sqrt{\beta}\end{array}\right) \cdot\left(\begin{array}{l}x \\ x^{\prime}\end{array}\right)$

Here, $x$ is the transverse, horizontal displacement and $x^{\prime}$ is the gradient of $x$ with respect to the longitudinal position (distance along the reference trajectory). $x_{N}$ and $x_{N}^{\prime}$ are the corresponding co-ordinates in the normalised phase space, and $\alpha$ and $\beta$ are Twiss parameters. The evolution of the Twiss parameters along a beamline is determined by the design of the beamline, once the values at the start of the beamline are specified.

A similar transformation to Eq. (1) applies to the vertical displacement $y$. However, in the absence of coupling, no new features appear when considering the vertical dynamics; hence, we consider only the horizontal phase space in this paper.

We consider the idea of beam tomography in normalised phase space. This is distinct from beam tomography in real phase space, since transformations along a beamline involving linear elements (drifts, dipoles, quadrupoles) can be represented purely as rotations in normalised phase space, with a rotation angle equal to the betatron phase advance. Tomography involves obtaining projections of the object to be imaged from a range of different angles [10]. We shall demonstrate that for beam phase space tomography, 
the projection angles are exactly equal to the rotation angles in normalised phase space. In real phase space, the projection angles are different from those in normalised phase space.

In practice, tomography is carried out in real phase space. This involves first choosing a location on the beamline at which to reconstruct the phase space distribution of the beam. In order to perform the tomography, we need to know the projection of this distribution onto coordinate space, with the projection taken at different rotation angles of the distribution. With fixed magnet strengths and locations, a screen at a fixed position provides a projection at a single angle. Ideally, we would like to have projections for many angles at uniform intervals that cover a full range from $0^{\circ}$ to $180^{\circ}$, in order to have a good sampling of the distribution. In practice, if screens are used (instead of, say, quadrupole scans), there may be only a few angles. The screens are often designed to be at intervals of equal betatron phase advances, which means uniform intervals of angles in normalised phase space. Therefore, the angles in real phase space may not be uniform.

The decision to choose uniform intervals of angles in normalised phase space rather than in real phase space already has implications for PITZ [12], ALICE [13] and the FLASH [14]. The design of the tomography sections in these machines means that projections at equal intervals of angles in normalised phase space are obtained, as long as matched Twiss parameters are used. However, the reconstruction has so far been carried out in real phase space. This choice of uniform angles in one space, and reconstruction in another, is the object of our study. This issue is also relevant to other rotation methods, such as quadrupole or solenoid scans. Beam phase space tomography using these techniques has also so far only been carried out in real phase space $[1,3]$. However, it is possible to perform a consistent analysis in normalised phase space simply by making an appropriate choice of magnet currents.

In Section 2, we review the method for phase space tomography. In Section 3, we develop the method for tomography in normalised phase space. In Section 4, we discuss the impact on the ALICE beamline and the results of reconstructions in different spaces. In Section 5, we consider how tomography in normalised phase space may be used to improve the reconstruction. Section 6 then concludes with a summary of the main results.

\section{Phase space tomography}

In this section, we shall provide a brief review of the standard beam tomography method, based on Ref. [9]. The presentation of this pictorial view will make it simpler to develop the normalised phase space picture in the next section.

In a typical set-up for beam tomography, there is a section of the beamline in which the phase space distribution is subject to a known transformation, that can be controlled in some way. If there is a quadrupole, we can vary its strength. If there are a number of screens, each screen shows the projection of the distribution after transformation through a different set of drift spaces and quadrupoles. Suppose that this section is between positions 1 and 2 on the beamline, and that the particles travel in the direction 1-2. Suppose there is a screen at 2, or some other means of capturing an image of the transverse beam distribution. The objective of this set-up is to measure the phase space distribution at 1 , the reconstruction location.

Consider the horizontal ( $x$ direction) phase space. From the distribution at position 1, we may calculate the distribution at the screen at position 2 . Assuming only uncoupled, linear dynamics, the $2 \times 2$ transfer matrix from positions 1 to 2 may be constructed from the transfer matrices for the components (drift spaces and quadrupoles) between positions 1 and 2 . Nonlinear effects may

arise from large oscillation amplitudes and space charge, but these are neglected in this study. The phase space co-ordinates at positions 1 and 2 are related to the transfer matrix $R$ by

$$
\left(\begin{array}{l}
x_{2} \\
x_{2}^{\prime}
\end{array}\right)=\left(\begin{array}{ll}
R_{11} & R_{12} \\
R_{21} & R_{22}
\end{array}\right) \cdot\left(\begin{array}{l}
x_{1} \\
x_{1}^{\prime}
\end{array}\right) .
$$

Reconstruction of the phase space distribution at position 1 can be carried out using a standard method [9]. The basic technique, as with any tomography procedure, is to find the projections on one axis (in this case, the $x$ axis) corresponding to projections at a range of different angles in the two-dimensional space (in this case, $x-x^{\prime}$ phase space). From the set of projections, the full twodimensional distribution may be reconstructed.

The procedure for finding a single projection for a particular angle (determined by the transformation between positions 1 and 2) consists of the following steps:

1. Calculate the projection at position 2 .

2. Calculate the angle of the corresponding projection at position 1 .

3. Calculate the distance scaling of the projection at position 1.

4. Calculate the projection at position 1 .

In step 1 , we need to find the projection onto the $x$ axis of the $x-x^{\prime}$ phase space distribution at position 2 . The projection at each value of $x$ is equal to the integral of the distribution along the $x^{\prime}$ direction, at that value of $x$. If we repeat this for different values of $x$, we obtain a set of projections. This set of numbers is called the projection along the $x$ direction. Although we do not know what this distribution is, the same result can be obtained from the real $x-y$ coordinate space distribution, obtained from the image on the screen at position 2 . If we integrate the image intensity along $y$ at each value of $x$, the result is the same as the projection in the phase space, since in each case we are effectively counting the number of particles within a narrow range of $x$ coordinate values.

In step 2, to obtain the angle of the corresponding projection at position 1, consider the schematic diagrams of the phase spaces in Fig. 1. The distribution changes shape from positions 1 and 2 under the transformation of Eq. (2). Since the transformation is linear, a straight line will remain a straight line; however, the angle of the line may change. At position 2, the projection is obtained by calculating a series of line integrals parallel to the direction P'Q' in Fig. 1(b). At position 1, these correspond to a series of line integrals parallel to the line PQ in Fig. 1(a). Following the convention in Ref. [10] and calling the line PQ a ray, we can see that the projection at position 1 (which is perpendicular to the ray) is along the direction $\rho$, with a corresponding angle $\theta$. Applying Eq. (2) to the points $\mathrm{P}$ and $\mathrm{Q}$, this angle can be derived: $\tan \theta=\frac{R_{12}}{R_{11}}$

a

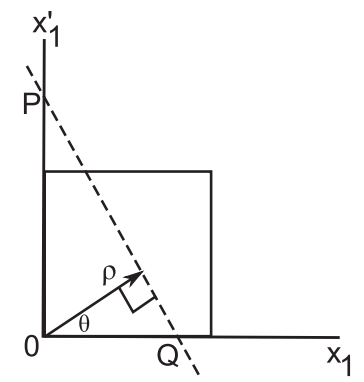

Fig. 1. (a) Schematic phase space distribution at the reconstruction location, and (b) at a screen. 
Step 3 involves finding $\rho$. By applying Eq. (2) to triangle POQ we find that

$\rho=\frac{x_{2}}{S}$

where

$S=\sqrt{R_{12}^{2}+R_{11}^{2}}$.

Note that $R_{11}$ is dimensionless and $R_{12}$ has the unit of metres. This means that Eqs. (3) and (5) that are used in phase space tomography [5] are dimensionally inconsistent. They are applied by treating $R_{12}$ as the number of metres, without the unit. To see that this is so, consider Fig. 1(a). In this phase space, the projections are taken at different angles. In order for these angles to be properly defined, a unit of $x$ must be equal to a unit of $x^{\prime}$. However, $x$ has the unit of metres and $x^{\prime}$ is dimensionless. So in this figure, $x$ must be treated as the number of metres, and not as the length. In this way, both $x$ and $R_{12}$ are redefined as dimensionless numbers, so that Eqs. (3) and (5) are now consistent. If other units of length are used instead of metres, the reconstructed distribution would appear stretched in the $x$ direction, and can simply be rescaled to metres after reconstruction. This is the way the equations are normally written and used in phase space tomography literature, e.g. $[5,9]$. One way to make the equations dimensionally consistent is to replace $R_{12}$ by $k R_{12}$, where $k$ is a constant factor. If the unit of $R_{12}$ is metres, then $k=1 \mathrm{~m}^{-1}$. If the unit of $R_{12}$ is millimetres, then $k=1 \mathrm{~mm}^{-1}$. In this way, the equations would become dimensionally consistent.

For step 4, suppose that the projection obtained in Fig. 1(b) is $p\left(x_{2}\right)$. The corresponding projection in Fig. 1(a) is $P_{\theta}(\rho)$. The total number of particles is the integral of either of these projections. The integrals must be equal:

$\int P_{\theta}(\rho) d \rho=\int p\left(x_{2}\right) d x_{2}$

Applying Eq. (4) to this, we find the projection at position 1

$P_{\theta}(\rho)=s p\left(x_{2}\right)$.

The images recorded from a screen at position 2 (with some adjustment of the beamline between positions 1 and 2) can thus be processed to give the projections $P_{\theta}(\rho)$ at the reconstruction location at position 1 , for a range of $\rho$ and $\theta$. Once this is achieved, we have the same problem as that, for example, of X-ray tomography in medical imaging. The image can be reconstructed using a standard method for real space tomography.

In principle, any reconstruction technique may be used. In practice, some techniques may be more suited to particular conditions. The Filtered Back Projection (FBP) is often used when a large number of angles are available, such as in quadrupole scans [5]. If there are a few angles, streaking artefacts would show up on the reconstructed image along the direction of the angles. The Maximum Entropy Technique (MENT) is useful when there are only a few angles, possibly obtained from a few screens with drift spaces in between [2]. If there is a large number of angles, the computation time would be long and convergence may be difficult. The Algebraic Reconstruction Technique (ART) [8] could in principle be used in either case, but tends to be noisy [10]. These are just a few examples.

The effect of the distribution of angles on reconstruction is likely to be different for different techniques, because of the different ways that the projection data are processed. In FBP, if some angular intervals are smaller, then there could be greater intensity and hence distortion along corresponding directions, since the technique involves a sum of the back projections over all angles [10]. In MENT, larger intervals for some angles could give less accurate results along the corresponding directions, because there would be fewer constraints along those directions [11]. In this paper, we use the FBP technique because it is simple to implement, and it allows us to demonstrate both the effect of a few angles, and of many angles. We have written a Matlab code using the FBP method [10] for this purpose. The effect on MENT will be explored in a future paper.

From Ref. [10], the formula for the FBP technique is

$f\left(x, x^{\prime}\right)=\frac{\pi}{K} \sum_{i}^{K} Q_{\theta_{i}}\left(x \cos \theta_{i}+x^{\prime} \sin \theta_{i}\right)$

where

$Q_{\theta}(\rho)=\int_{-\infty}^{\infty} S_{\theta}(w)|w| e^{j 2 \pi w \rho} d w$

is the filtered projection, $S_{\theta}(w)$ being the Fourier transform of the projection $P_{\theta}(\rho)$. The summation is a discretisation of an integral over angles, and the factor $\pi / K$ is the uniform interval of $K$ uniformly spaced angles $\theta_{i}$. In the case of nonuniform intervals, $\pi / K$ becomes the average interval. If some angles are more closely spaced, there would be more terms in the summation for those angles. As a result, we may expect $f\left(x, x^{\prime}\right)$ to become higher in the corresponding directions and give rise to distortion.

Eq. (8) is a discretisation over angles of the integral [10]

$f\left(x, x^{\prime}\right)=\int_{0}^{\pi} Q_{\theta}\left(x \cos \theta+x^{\prime} \sin \theta\right) d \theta$.

If the intervals in angles are not uniform, the discretisation may be rewritten as

$f\left(x, x^{\prime}\right)=\sum_{i}^{K} Q_{\theta_{i}}\left(x \cos \theta_{i}+x^{\prime} \sin \theta_{i}\right) \Delta \theta_{i}$

$\Delta \theta_{i}$ may be treated as a weighting factor. If the angular intervals on two sides of a projection are different, the choice of $\Delta \theta_{i}$ is not obvious. A simple approach would be to consider a linear interpolation of the projections in between angles, and then apply the trapezium rule to carry out the integration. It can be then shown that $\Delta \theta_{i}$ is given by the sum of half of the angular interval on one side of projection $i$, and half of the interval on the other side. We shall call the product of $\Delta \theta_{i}$ and the projection a weighted projection.

\section{Normalised phase space}

Reconstruction in normalised phase space can be done with a simple extension of the method given in Section 2. The matrix in Eq. (2) transforms the initial distribution in real phase space to the distribution at the screen. Based on this matrix, the procedure in Section 2 reconstructs the initial distribution.

The initial distribution may be considered the result of the transformation of the distribution in normalised phase space to real phase space. The transformation is given by the inverse of Eq. (1). In order to reconstruct in normalised phase space, we only need to replace the matrix in Eq. (2), by a matrix that transforms the distribution all the way from the normalised phase space to the distribution at the screen. This matrix is simply a product of the matrix in Eq. (2), and the matrix that transforms from normalised to real phase space. The latter matrix may be obtained by inverting equation (1) as follows:

$\left(\begin{array}{c}x \\ x^{\prime}\end{array}\right)=\left(\begin{array}{cc}\sqrt{\beta_{1}} & 0 \\ -\frac{\alpha_{1}}{\sqrt{\beta_{1}}} & \frac{1}{\sqrt{\beta_{1}}}\end{array}\right) \cdot\left(\begin{array}{c}x_{N} \\ x_{N}^{\prime}\end{array}\right)$

where the subscript 1 means that the Twiss parameters refer to position 1 . The matrix on the right-hand side is the required matrix. Inserting this into the right-hand side of Eq. (2) gives the 
new transfer matrix $\tilde{R}$ needed for the reconstruction in normalised phase space:

$\tilde{R}=\left(\begin{array}{ll}R_{11} & R_{12} \\ R_{21} & R_{22}\end{array}\right) \cdot\left(\begin{array}{cc}\sqrt{\beta_{1}} & 0 \\ -\frac{\alpha_{1}}{\sqrt{\beta_{1}}} & \frac{1}{\sqrt{\beta_{1}}}\end{array}\right)$

The procedure in Section 2 that is described after Eq. (2) may then be followed.

We now demonstrate that the projection angle $\theta$ in the normalised phase space is equal to the phase advance $\mu$. This can be done using the relation between the transfer matrix and the Twiss parameters at positions 1 and 2:

$\left(\begin{array}{ll}R_{11} & R_{12} \\ R_{21} & R_{22}\end{array}\right)=\left(\begin{array}{cc}\sqrt{\beta_{2}} & 0 \\ -\frac{\alpha_{2}}{\sqrt{\beta_{2}}} & \frac{1}{\sqrt{\beta_{2}}}\end{array}\right) \cdot\left(\begin{array}{cc}\cos \mu & \sin \mu \\ -\sin \mu & \cos \mu\end{array}\right) \cdot\left(\begin{array}{cc}\frac{1}{\sqrt{\beta_{1}}} & 0 \\ \frac{\alpha_{1}}{\sqrt{\beta_{1}}} & \sqrt{\beta_{1}}\end{array}\right)$

where the subscript 2 means that the Twiss parameters refer to position 2. We can understand the right-hand side in a simple way; the distribution at screen 1 is transformed to normalised phase space, propagated to screen 2 by a rigid rotation through angle $\mu$, and transformed back to real phase space. Substituting this into Eq. (13), we find

$\tilde{R}=\left(\begin{array}{cc}\sqrt{\beta_{2}} & 0 \\ -\frac{\alpha_{2}}{\sqrt{\beta_{2}}} & \frac{1}{\sqrt{\beta_{2}}}\end{array}\right) \cdot\left(\begin{array}{cc}\cos \mu & \sin \mu \\ -\sin \mu & \cos \mu\end{array}\right)$.

We can now apply Eq. (3) to this matrix to find $\theta$. Note that the original transfer matrix $R$ in Eq. (2) has been changed to $\tilde{R}$ defined in Eq. (13). So $R_{11}$ and $R_{12}$ in Eq. (3) must also be replaced by the elements in the first row of Eq. (13). These are equal to those in the first row of Eq. (15), which are $\sqrt{\beta_{2}} \cos \mu$ and $\sqrt{\beta_{2}} \sin \mu$. Substituting these into Eq. (3) for $R_{11}$ and $R_{12}$, respectively, we find

$\tan \theta=\tan \mu$.

So $\mu$ is indeed the projection angle.

At this stage, we emphasise that the significant result is that if the tomographic reconstruction is performed without a normalising transformation, then the projection angles need to be calculated from the transfer matrices; they are not simply the betatron phase advances. This is significant for tomography in accelerator beamlines, which are often designed with uniform betatron phase advance between successive screens, i.e. uniform distribution of projection angles in normalised phase space $[12,14,17]$. The distribution of angles in real phase space will not necessarily be uniform, and unless this is taken into account, some distortion in the reconstructed image may result.
To illustrate this point, consider the corresponding rays in real and normalised phase spaces shown in Fig. 2. (The projection direction is perpendicular to the ray.) Fig. 2(a) shows a Gaussian distribution in real phase space, with rays that are at uniform angular intervals. In normalised phase space, some of the intervals become smaller, whereas others become larger, as shown in Fig. 2(b). This suggests that reconstruction of the image in normalised phase space may lead to a distorted image, because of the uneven sampling.

Fig. 3 illustrates the effect of the opposite transformation-starting with uniform intervals of angles in normalised phase space, shown in Fig. 3(a). This results in a nonuniform distribution of rays in real phase space, shown in Fig. 3(b). This could now lead to distortions in the reconstructed image, if the reconstruction is performed using an algorithm that assumes uniform distribution of projection angles. This is the situation that applies to the tomography sections in accelerators.

For tomography sections that are designed with screens at uniform phase advance intervals, the projection angles will be at uniform intervals in normalised phase space. Reconstructions are usually carried out in real phase space. As we have seen in Fig. 3, this could lead to distorted images. On the other hand, notice that many of the rays get bunched together into a smaller range of angles in Fig. 3(b). This suggests that optimisation of the design of a tomography section in an accelerator beamline may best be done by considering the expected distribution of the beam in normalised phase space. This could be important where the number of projections may be limited, e.g. by a limited number of screen locations.

Note that the evolution of the Twiss parameters along an uncoupled linear beamline is such that a beam distribution in normalised phase space will perform a rigid rotation as the beam moves along the beamline. In that sense, construction of the distribution in normalised phase space presents a more global picture of the beam properties, than the representation of real phase space distribution at a particular point in the lattice.

Fig. 3(a) shows a circular distribution. This is typical of a distribution transformed to normalised phase space, if the distribution is correctly matched to the lattice. Such a distribution will be invariant under linear transport along an uncoupled beamline (as long as the lattice functions are calculated correctly). More generally, a given distribution will perform a rigid rotation in normalised phase space. Note that it is not necessary to know the matched lattice functions (i.e. the Twiss parameters describing a transformation that makes the beam distribution in phase space a perfect circle) in order to perform tomography in

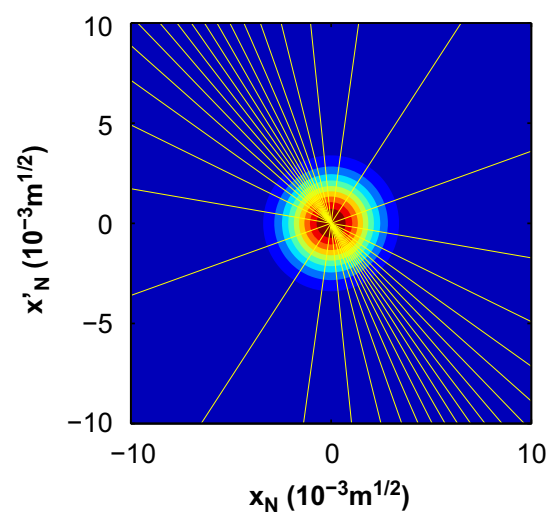

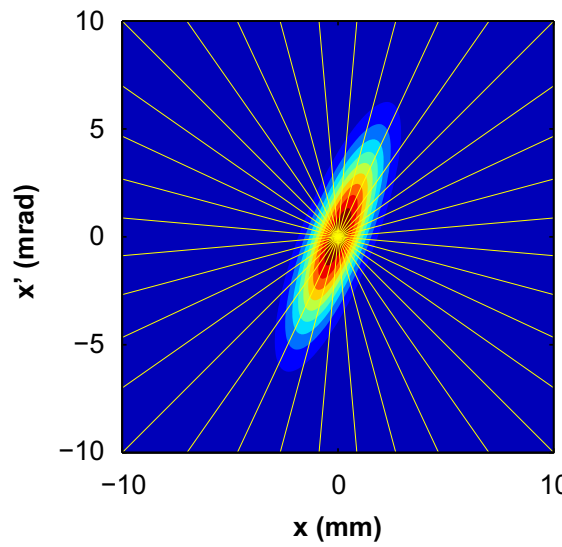

Fig. 2. (a) Real phase space with rays at uniform angular intervals. (b) Normalised phase space. 
a

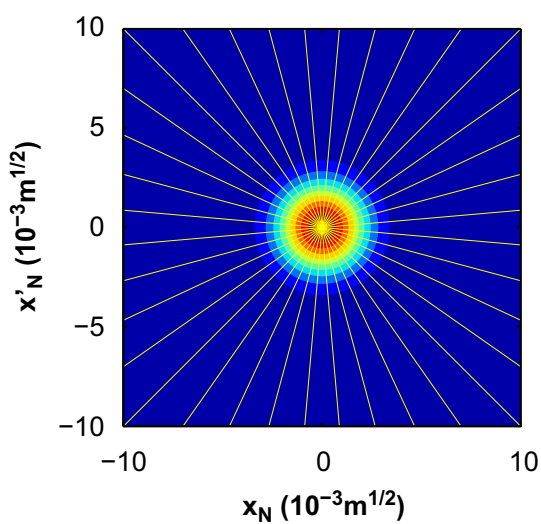

b

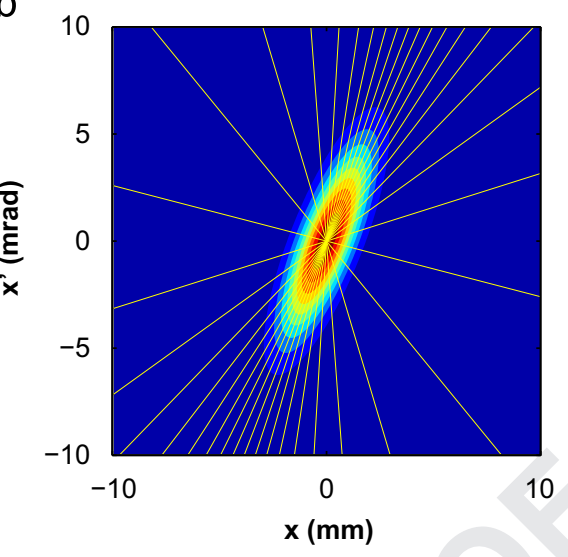

67

Fig. 3. (a) Normalised phase space with rays at uniform angular intervals. (b) Real phase space. normalised phase space; any Twiss parameters can be used to perform the normalising transformation, but it should be remembered that the projection angles will depend on the choice of Twiss parameters. If we do not know the matched lattice functions, but wish to obtain a circular distribution, we could estimate the Twiss parameters from the reconstructed image in real phase space, as explained in Section 5.

\section{ALICE tomography section}

The ALICE (Accelerators and Lasers In Combined Experiments) accelerator [15] is used as an injector to the EMMA accelerator [16]. The injection line incorporates a tomography diagnostic section which has been modified to operate at full ALICE energy (35 MeV), albeit in single bunch mode. After the main ALICE linac there is a dipole magnet which extracts the beam from the ALICE accelerator as part of the dog leg at the start of the EMMA injection line.

The layout of the ALICE tomography section is shown in Fig. 4. The tomography section consists of two consecutive FODO cells, with an Yttrium Aluminium Garnet (YAG) screen at either end, and a further screen at the mid point. Immediately before the tomography section is a matching section of four quadrupole magnets. The design of the injection line, and of the tomography section in particular, is based on the collection of tomography data at the three screens, with the quadrupole strengths fixed for a phase advance of $60^{\circ}$ from one screen to the next. If the beam distribution is correctly matched to the Twiss parameters, then the images on all three screens will be identical.

The screens are labelled as 1-3 in Fig. 4. The electron beam properties have been modelled in Refs. [13,17]. From recent measurements using the slit-scan technique [18], the emittance is known to be in the range of $5-10 \mathrm{~mm}$ mrad. In our simulation, we assume a value of $5 \mathrm{~mm}$ mrad. The Twiss parameters are the same at all three screens: $\alpha=-1.235$ and $\beta=0.7439 \mathrm{~m}$. These values have been determined to match the expected beam distribution, based on modelling in ASTRA [19] of the beam distribution produced from the electron gun, and propagated to the tomography section [20]. It has also been shown [21] using ASTRA and GPT [22] that in the ALICE tomography section, the space charge effect is not significant for an electron bunch with a charge of $10 \mathrm{pC}$ and an energy of $10 \mathrm{MeV}$. We assume in this paper that the bunch charge is small enough for the space charge effect to be neglected.

The transfer matrix from screens 1 to 2 is obtained from the drift lengths and quadrupole magnet strengths between the

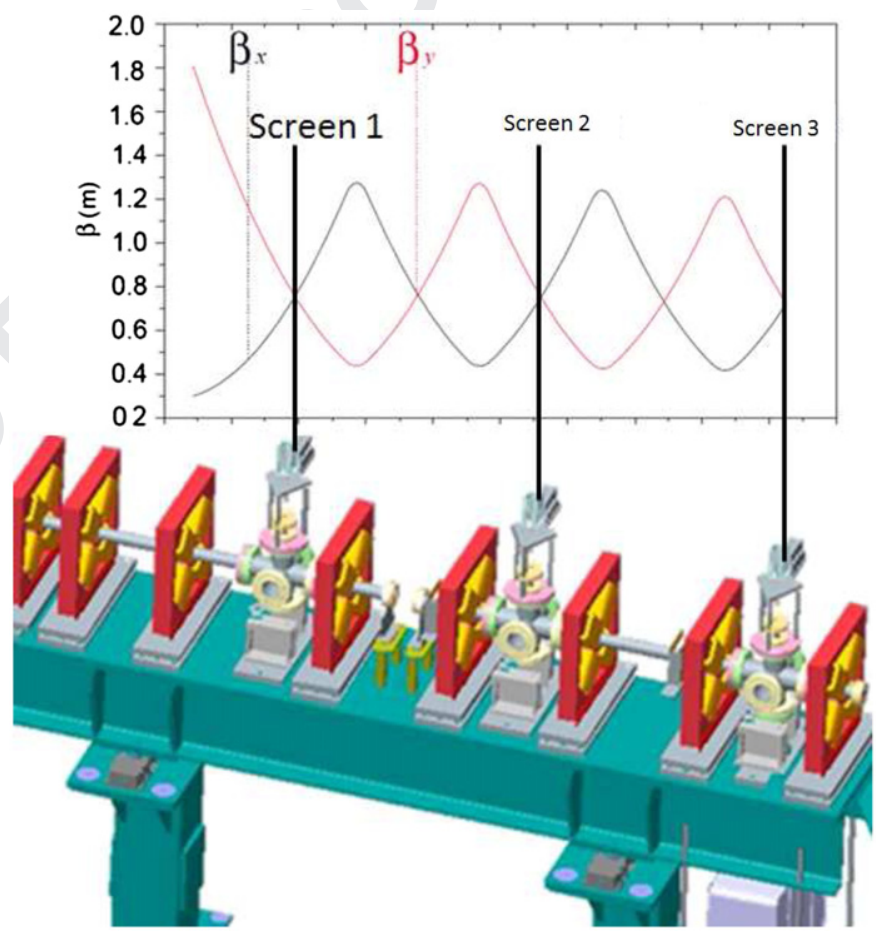

Fig. 4. The ALICE tomography section.

screens, and is given by

$R\left(S_{2}, S_{1}\right)=\left(\begin{array}{cc}-0.5695 & 0.6442 \mathrm{~m} \\ -2.9398 \mathrm{~m}^{-1} & 1.5695\end{array}\right)$.

Since the section from screens 2 to 3 is identical to that from screens 1 to 2 , the transfer matrix from screens 1 to 3 is given by

$R\left(S_{3}, S_{1}\right)=R\left(S_{3}, S_{2}\right) \cdot R\left(S_{2}, S_{1}\right)=R\left(S_{2}, S_{1}\right)^{2}$.

From the Twiss parameters at screen 1, a simulation of a matched Gaussian distribution is shown in Fig. 2(a). The projections at all three screens can then be calculated analytically using the above matrices. Following the procedure in Section 2, the phase space distribution at screen 1 is then reconstructed. The results are shown in Fig. 5. Fig. 5(a) is obtained using Eq. (8), with projections that are not weighted. The streaking artefacts are clear. Note that although the projection angles are uniformly spaced in normalised phase space (corresponding to the $60^{\circ}$ phase advance across each FODO cell in the tomography section), 
a

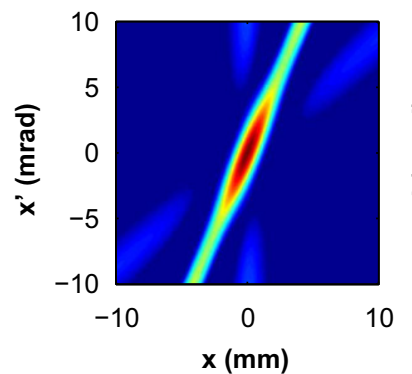

b

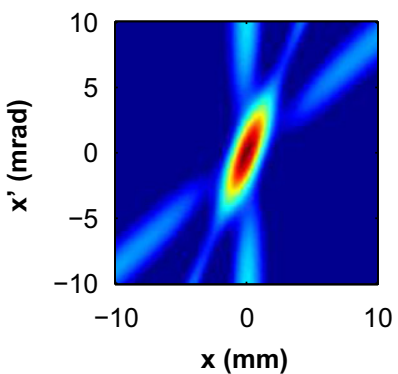

C

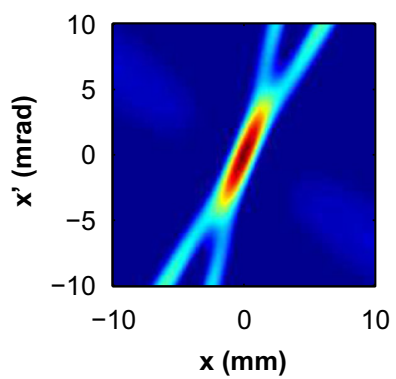

67 tomography section consisting of a drift space with 18 screens. The positions of these screens are chosen to correspond to projection angles in normalised phase space from $-85^{\circ}$ to $85^{\circ}$, at intervals of $10^{\circ}$. This means that the reconstruction location is chosen to be right at the centre of the array of screens. In order to determine the positions of the screens, the transfer matrix for a drift space is used in Eq. (13) to give

$\tilde{R}=\left(\begin{array}{ll}1 & L \\ 0 & 1\end{array}\right) \cdot\left(\begin{array}{cc}\sqrt{\beta} & 0 \\ -\frac{\alpha}{\sqrt{\beta}} & \frac{1}{\sqrt{\beta}}\end{array}\right)$

where $L$ is the length of the drift space. The Twiss parameters here refer to the position of the screen. We redefine $L$ as the displacement of each screen from the centre of the array of screens, where the phase space distribution is to be reconstructed. This means that $L$ can be negative, which gives the correct transfer matrix when the screen is before the reconstruction location. We can then solve for $L$ for each screen using Eq. (3).

Assuming the same distribution as before, the projections at each screen may again be computed analytically. The reconstruction is carried out in real phase space. The result using Eq. (8) with projections that are not weighted is shown in Fig. 7(a). Compared with Fig. 2(a), there is a clear elongation of the reconstructed image. This is not a consequence of the artefacts arising from a limited number of projection angles with the FBP tomography algorithm, but a result of performing tomography in real phase space with a uniform distribution of angles in normalised phase space. Fig. 3(a) shows that in this case, the rays are more closely spaced around the major axis of the elliptical distribution. This produces the elongated distortion.

Next, consider what happens if we use uniform angular intervals in real phase space. To find the corresponding screens positions, replace Eq. (19) by the just the transfer matrix for

In order to illustrate more clearly the effects of reconstructing in a different space, we consider a simple, hypothetical case in which a more complete range of angles is available. This will 
a

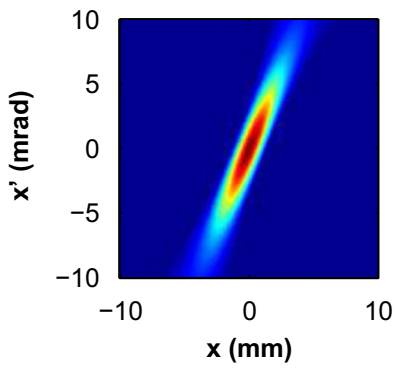

b

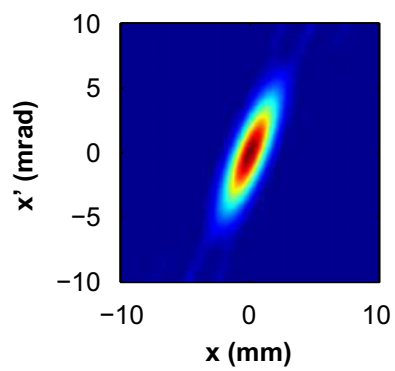

C

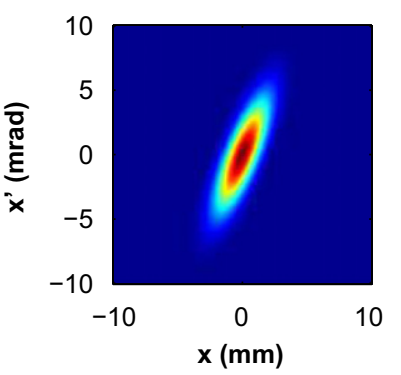

67

7. (a) Reconstruction in real phase space with projections not weighted, using 18 uniform angles in normalised phase space. (b) Reconstruction in real phase spac using 18 uniform angles in real phase space. (c) Reconstruction in real phase space with weighted projections, using 18 uniform angles in normalised phase space.

a

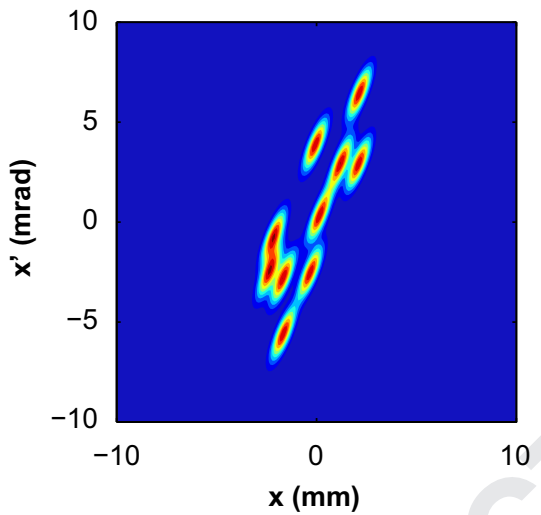

b

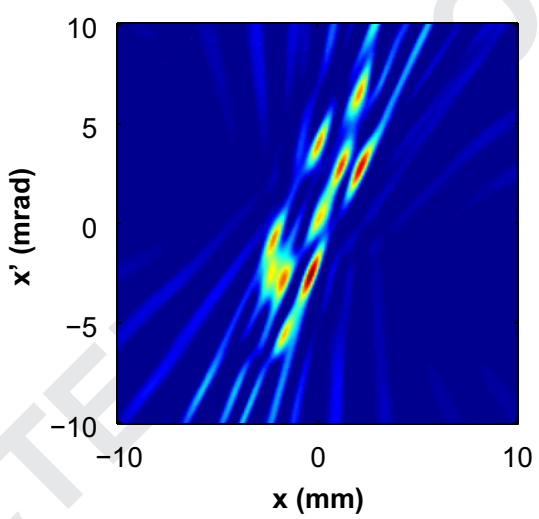

Fig. 8. (a) Distribution in real phase space. (b) Reconstruction in real phase space.

a drift space,

$R=\left(\begin{array}{ll}1 & L \\ 0 & 1\end{array}\right)$

and solve for $L$ for each screen using Eq. (3). The reconstruction result is shown in Fig. 7(b). This reproduces the correct distribution with no elongation, as we would expect. Next, the reconstruction is carried out using Eq. (11), with projections that are weighted. The result is shown in Fig. $7(\mathrm{c})$. This reproduces the correct distribution, confirming that using Eq. (11) with the weighted projections is valid.

\section{Improving the reconstruction}

Beam distributions are often more complex than simple Gaussians. We consider a more complex hypothetical case where the distribution is made up of a group of closely spaced Gaussian spots, as shown in Fig. 8(a). This provides a test of the ability of a reconstruction method to resolve the spots. Note that each spot has a circular distribution in normalised phase space. To transform the distribution to real phase space, we use the Twiss parameters from the ALICE tomography section. We continue to use the hypothetical system of 18 screens described in Section 4, and start with the case of equal angular intervals in real phase space. The projections from the screens are used to reconstruct Fig. 8(a). The result is shown in Fig. 8(b). The spots are all reproduced and at the correct positions. However, the resolution is less clear.

We then look at the case of equal angular intervals in normalised phase space. When Fig. 8(a) is transformed to normalised phase space, the distribution is as shown in Fig. 9(a). Note that the screens would now be at different positions from the previous case. When we use the projections from these screens to reconstruct the distribution in normalised phase space, we get Fig. 9(b). This time, the spots are clearly reproduced. The obvious step to transform the co-ordinates to real phase space gives Fig. 10. This is much clearer than Fig. 8(b). Apart from the faint artefacts, the spots look almost the same as the original Fig. 8(a).

Reconstruction in normalised phase space relies on the assumption that the Twiss parameters are close to the design values. Only then will phase advances between screens be uniform. In practice, actual Twiss parameters could be quite different, because of inaccuracies in the beam model, and because of beamline errors not known at the time of design. The result is that the phase advances will no longer be equal, so the projection angles will not be uniform in normalised phase space. However, note that it is not necessary to know the matched Twiss parameters at the start of the tomography section: any values can be used. We could try the design values or we could estimate from the reconstructed image in real phase space, as described below. But given some initial values for the Twiss parameters, an accurate knowledge of the phase advance from the start of the tomography section to a screen used for capturing the beam profile will depend on the accuracy of knowledge of the strengths and positions of the magnets between the start of the section and the screen. Generally, the number of magnets will be rather small, so it should be possible, with sufficient effort, to achieve the accuracy in alignment and strength calibration needed to ensure reliable results from tomography measurements.

We have not discussed beam tomography using other ways to change the projection angle, such as a quadrupole scan. This is a simple way to get a good reconstruction, if a nearby quadrupole is available, and the current control and image capture system have been set-up. When the quadrupole strength is varied, its transfer matrix is changed. This changes the projection angle at a reconstruction location that is on the opposite side of the quadrupole from the screen (in this case we may need only a single screen). 
a

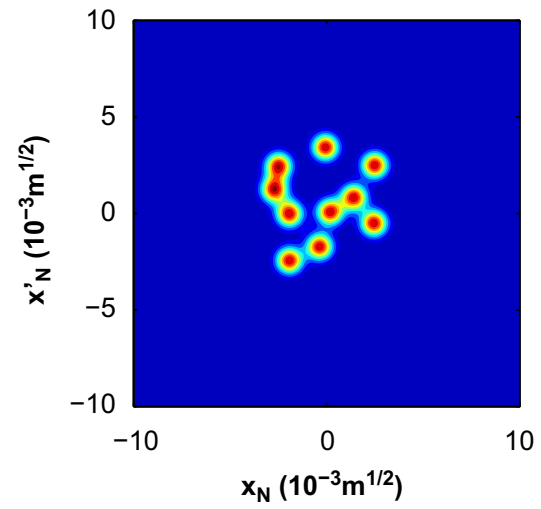

b

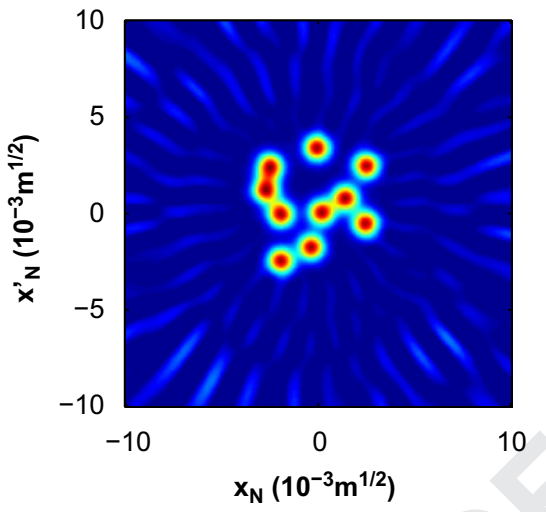

Fig. 9. (a) Distribution in normalised phase space. (b) Reconstruction in normalised phase space.

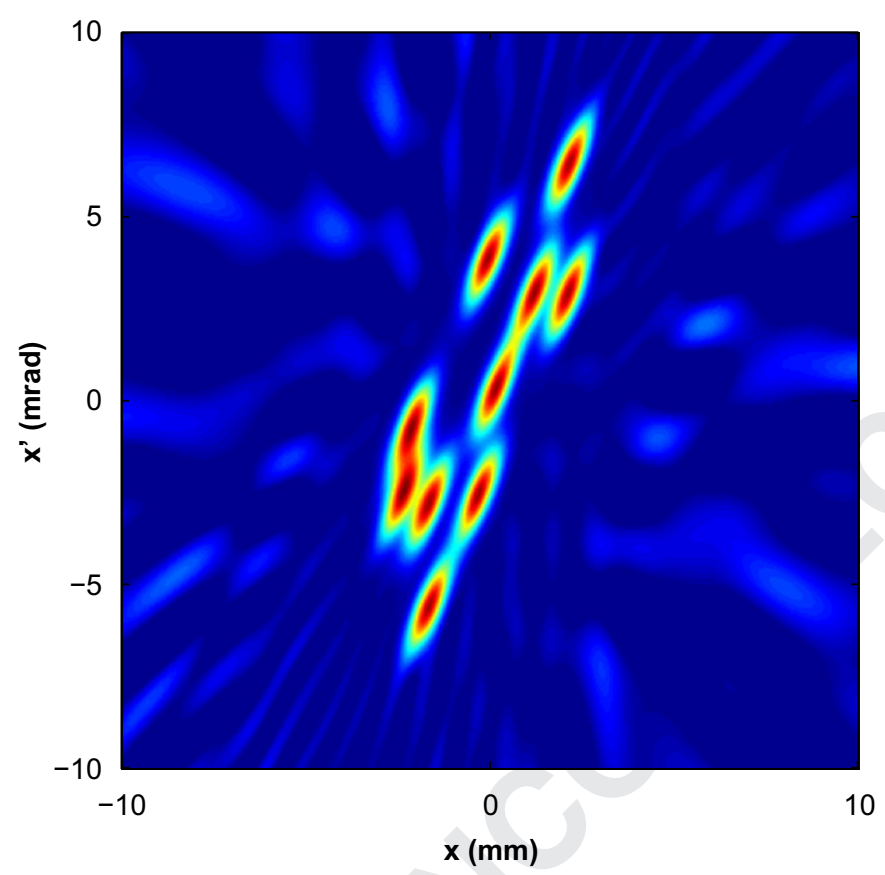

Fig. 10. Distribution obtained by transforming the co-ordinates in the reconstruction in normalised phase space to the real phase space.

Since the quadrupole strength can usually be adjusted continuously up to some maximum, there is in principle no restriction on the number of angular intervals for which projections can be obtained. By selecting the right intervals of current, the angles can be chosen to be uniform in either the real or the normalised phase space, and reconstruction carried out in the appropriate space.

Although any Twiss parameters can be used in the normalising transformation, there could be certain advantages for a phase space in which the distribution is roughly circular. One example is Fig. 10, which appears to have a better resolution than Fig. 8(b). This can be achieved with the following procedure:

1. Carry out tomography in real phase space to make an initial estimate of the Twiss parameters that describe the real beam distribution at the reconstruction point.

2. Using these Twiss parameters, calculate the magnet strengths that would increase the phase advance between the reconstruction point and the screen in uniform steps.

3. Take the screen images with the magnet strengths calculated in step 2, and perform the tomography in normalised phase space (using the Twiss parameters from step 1 for the normalising transformation).

If the results from step 1 are reasonably accurate, this should give a distribution that appears roughly circular in normalised phase space.

\section{Conclusion}

We have discussed the reconstruction of a distribution in normalised phase space, and proposed a method for carrying out such a reconstruction.

We have suggested an approach for applying the FBP algorithm to projections with nonuniform intervals of angles. This approach multiplies each projection with a suitable weight, before summing the filtered back projections. It is equivalent to approximating the projection at any angle with a linear interpolation over the measured projection, and then doing the exact integration of Eq. (11).

We show that this approach could remove the distortions that might arise from a direct sum of the filtered back projections, if the intervals of angles are not uniform. However, this only works when the intervals are small enough, and the distribution not too complex. We have shown that if there were only a few angles, or if the distributions were made up of many spots, then the use of the weighted projections could still produce poor quality reconstructions.

We have seen that the reconstruction in normalised phase space is less dependent on the number of angles. Even with only three angles, it is possible to produce a nearly circular image. This suggests that transforming the normalised co-ordinates to real phase space may be able to preserve the shapes better. We have applied this to a simple distribution using three angles, and a complex distribution with many spots. The reconstruction of both cases shows that the method is indeed able to produce images that are much closer to the original distributions, compared with a direction reconstruction in the real phase space.

Tomography in normalised phase space is already partially implemented in some accelerators, such as PITZ, ALICE and FLASH, in the sense that the tomography sections in these accelerators have been designed with screens at positions that correspond to uniform angular intervals in normalised phase space. However, the idea of reconstructing in normalised space has not so far been exploited. It is interesting to note that the research group at PITZ [12] has shown that when the screens are spaced at positions that would give equal phase advances between adjacent screens, the difference between reconstructed and original distribution is minimised. This is demonstrated using the Maximum Entropy Technique (MENT), instead of the FBP, and the reconstruction is 
carried out directly in real phase space. That there is a connection with this paper is clear for two reasons. One reason is that, as we have shown, the phase advance is equal to the projection angle in normalised phase space. The next reason is that the uniform intervals of angles in normalised phase space gives better reconstruction results, which we have also demonstrated using the FBP. The difference is that in our case, the FBP reconstruction must be carried out in normalised phase space first, and then transformed to real phase space. This could be due to the different ways that the two techniques process the projections data.

The range of accessible angles may be limited by the number of screens or, if quadrupoles are used, the quadrupole strengths. We have seen that when the rays from projections at uniform intervals of angles are transformed from normalised to real phase space, they can get bunched up in some directions, where the intervals of angles become much smaller. This suggests that we could sample the angles in these regions more finely. Then even if we leave out the other angles, we can still get a reasonable sample of angles in the normalised phase space, which we have shown is sufficient for good reconstruction. Thus, the transformation from normalised to real phase space provides the added flexibility of choosing the optimal range of angles.

Finally, it should be mentioned that as the distribution in normalised phase space is invariant (under linear transport) up to a rotation angle, it provides a global description of the phase space distribution along the whole beam line.

\section{Uncited reference}

Q1 [23].

\section{References}

[1] D. Stratakis, et al., Phase space tomography of beams with extreme space charge, in: Proceedings of PAC07, Albuquerque, New Mexico, USA, 2007.

[2] D. Reggiani, et al., Transverse phase-space beam tomography at PSI and SNS proton accelerators, in: Proceedings of IPAC10, Kyoto, Japan, 2010.
[3] F.E. Hannon, et al., Phase space tomography using the Cornell ERL gun, in: Proceedings of EPAC08, Genoa, Italy, 2008.

[4] V. Yakimenko, M. Babzien, I. Ben-Zvi, R. Malone, X.-J. Wang, Phys. Rev. Spec. Top. Acceler. Beams 6 (2003) 122801.

[5] D. Stratakis, R.A. Kishek, H. Li, S. Bernal, M. Walter, B. Quinn, M. Reiser P.G. O'Shea, Phys. Rev. Spec. Top. Acceler. Beams 9 (2006) 112801

[6] F. Zhou, A. Kabel, J. Rosenzweig, R. Agustsson, G. Andonian, D. Cline, A. Murokh, V. Yakimenko, Phys. Rev. Spec. Top. Acceler. Beams 9 (2006) 114201.

[7] D. Stratakis, K. Tian, R.A. Kishek, I. Haber, M. Reiser, P.G. O'Shea, Phys. Plasmas 14 (2007) 120703.

[8] D. Xiang, Y.-C. Du, L.-X. Yan, R.-K. Li, W.-H. Huang, C.-X. Tang, Y.-Z. Lin, Phys. Rev. Spec. Top. Acceler. Beams 12 (2009) 022801.

[9] C.B. McKee, P.G. O’Shea, J.M.J. Madey, Nucl. Instr. and Meth. Phys. Res. A 358 (1995) 264.

[10] A.C. Kak, M. Slaney, Principles of Computerized Tomographic Imaging, SIAM, Philadelphia, USA, 2001, pp. 60-75, 〈http://www.slaney.org/pct/pct-toc.html 〉.

[11] C.T. Mottershead, IEEE Trans. Nucl. Sci. NS-32 (1985) 1970.

[12] G. Asova, S. Khodyachykh, M. Krasilnikov, F. Stephan, K. Floettmann, D.J. Holder, B. Muratori, Design consideration for phase space tomography diagnostics at the PITZ facility, in: Proceedings of DIPAC 2007, Venice, Italy, 2007.

[13] B.D. Muratori, S.L. Smith, S.I. Tzenov, C. Johnstone, Injection and extraction for the EMMA NS-FFAG, in: Proceedings of EPAC08, Genoa, Italy, 2008.

[14] F. Loehl, Measurements of the transverse emittance at the VUV-FEL, DESY, Hamburg, 2005. 〈http://gan.desy.de/ floehl/DiplomaThesis/DESY-THE SIS_2005-014_gray.pdf $\rangle$.

[15] Y. Saveliev, et al., Recent developments on ALICE (accelerators and lasers in combined experiments) at Daresbury Laboratory, in: Proceedings of IPAC10, Kyoto, Japan, 2010

[16] R. Barlow, et al., Nucl. Instr. and Meth. A 624 (2010) 1.

[17] D.J. Holder, B.D. Muratori, Modelling the ALICE electron beam properties through the EMMA injection line tomography section, in: Proceedings of PAC09, Vancouver, Canada, 2009

[18] J. Garland, Characterisation and optimisation of the ALICE accelerator as an injector for the EMMA NS-FFAG, in: Proceedings of IPAC10, Kyoto, Japan, 2010.

[19] K. Floettmann, ASTRA (A Space charge TRAcking code) user manual, 2000. $\langle$ http://www.desy.de/ mpyflo $\rangle$.

[20] B.D. Muratori, Y.M. Saveliev, ALICE (ERLP) injector design, in: Proceedings of EPAC08, Genoa, Italy, 2008.

[21] R.T.P. D'Arcy, D.J. Holder, B.D. Muratori, J. Jones, Modelling of the EMMA NS-FFAG injection line using GPT, in: Proceedings of IPAC10, Kyoto, Japan, 2010.

[22] GPT (General Particle Tracer), 〈http://www.pulsar.nl/gpt/〉.

[23] M.G. Ibison, K.M. Hock, D.J. Holder, M. Korostelev, Development of tomographic reconstruction methods for studies of transverse phase space in the EMMA FFAG injection line, in: Proceedings of IPAC10, Kyoto, Japan, 2010.
41

43 